\title{
Immune Reconstitution Inflammatory Syndrome Associated with Pneumocystis Jirovecii Pneumonia and Cytomegalovirus Colitis in a Patient with Rheumatoid Arthritis
}

\author{
Natsuki Shima, Ayako Kokuzawa, Keisuke Saito, Yasuyuki Kamata, Takao Nagashima and
} Kojiro Sato

\begin{abstract}
:
A 68-year-old woman presenting with rheumatoid arthritis was admitted due to pancytopenia caused by methotrexate. Pneumocystis jirovecii pneumonia was diagnosed based on the abnormal shadows observed on chest computed tomography, the presence of serum $\beta$-D-glucan, and positive $P$. jirovecii-DNA results in a sputum analysis. Subsequently, after treatment with leucovorin and trimethoprim-sulfamethoxazole, lung consolidation was found to be aggravated, along with a rapidly increasing leukocyte count. In addition, cytomegalovirus colitis was diagnosed. Both conditions were associated with immune reconstitution inflammatory syndrome caused by recovery from leukopenia. The patient was successfully treated with intravenous methylprednisolone pulse therapy and ganciclovir.
\end{abstract}

Key words: disease-modifying anti-rheumatic drugs, leukopenia, methotrexate, myelosuppression, pancytopenia

(Intern Med Advance Publication)

(DOI: 10.2169/internalmedicine.7176-21)

\section{Introduction}

Immune reconstitution inflammatory syndrome (IRIS) is defined as an aggravated immune response generated against various pathogens during recovery of the immune system in patients infected with human immunodeficiency virus (HIV) and treated with antiretroviral therapy (1). The pathogenesis of IRIS remains unclear, but an imbalance in the Th1/Th2 cytokine levels has been partly attributed to its pathogenesis (2-4). A young age of the patients at the initiation of anti-HIV therapy, low $\mathrm{CD}^{+}$cell count, and low ratio of $\mathrm{CD}$ $4^{+} / \mathrm{CD}^{+}$cells are predictors of IRIS in HIV-positive patients (5).

IRIS can also occur in immunocompromised non-HIV patients, such as those who have undergone organ transplantation; those with connective tissue diseases, malignancy, and neutropenia; and women during the postpartum period $(2,6-8)$. Potential mechanisms that promote recovery from immunodeficiency include discontinuation or rapid tapering of glucocorticoids or immunosuppressants or both, withdrawal of or reduction in the effects of anti-tumor necrosis factor (TNF)- $\alpha$ antibodies, and the use of immunecheckpoint antagonists (6).

We herein report a patient with rheumatoid arthritis (RA) who developed IRIS associated with Pneumocystis jirovecii pneumonia (PCP) and cytomegalovirus (CMV) colitis immediately after discontinuation of methotrexate (MTX) because of pancytopenia.

\section{Case Report}

A 68-year-old woman presenting with a 20-year history of RA was admitted to our hospital because of pancytopenia development and the presence of an abnormal shadow on chest computed tomography (CT). She had been treated with MTX (6 mg; weekly) and actarit (100 mg; daily). Her vital signs on arrival in the hospital were as follows: body 


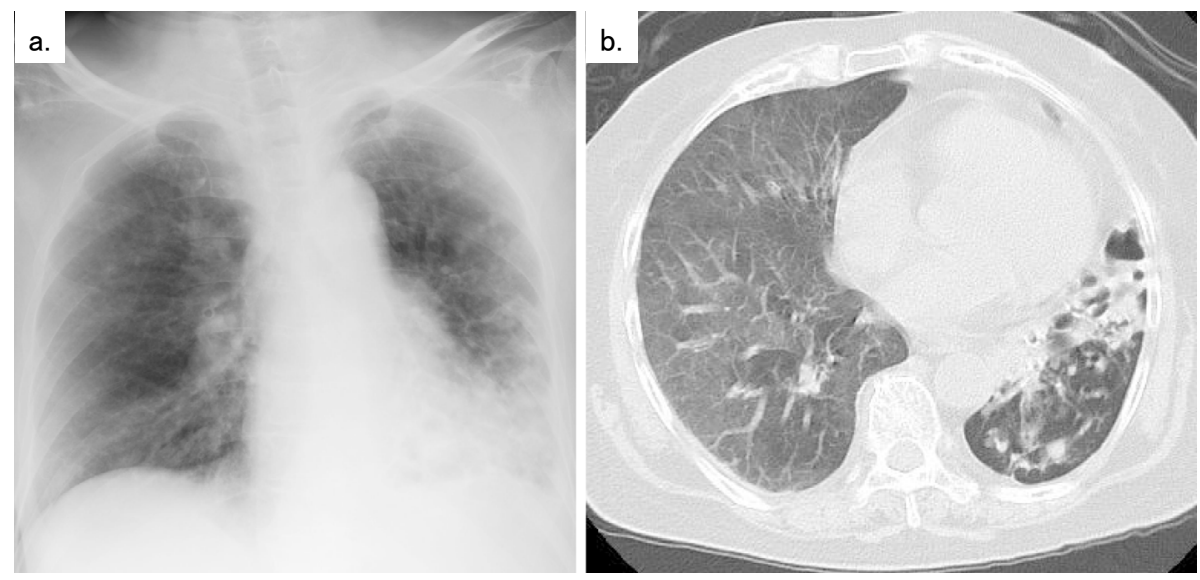

Figure 1. (a) Chest radiography and (b) chest computed tomography (CT) findings on admission. There was a ground-glass opacity in the right lung and consolidation in the left lower lobe.
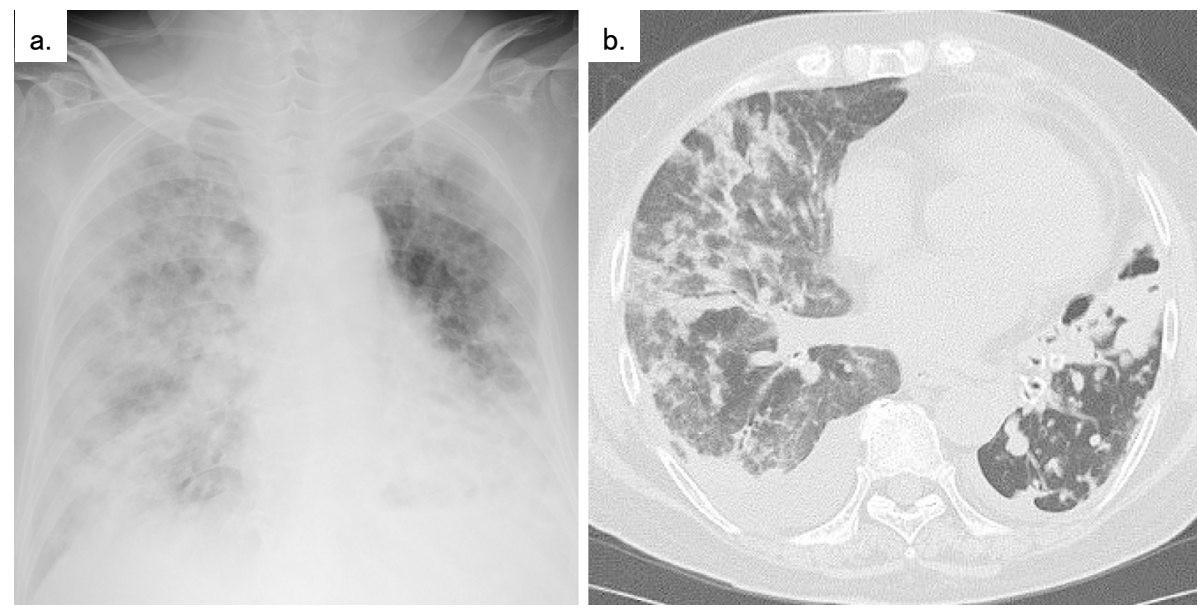

Figure 2. (a) Repeated chest radiograph and (b) chest CT on day 5 after admission. Consolidation and pleural effusion developed, particularly in the right lung.

temperature, $38.9{ }^{\circ} \mathrm{C}$; pulse rate, 122 beats/min; blood pressure, $104 / 58 \mathrm{mmHg}$; respiratory rate, 20 breaths/min; and percutaneous oxygen saturation, 95\% (2 L/min via nasal cannula). Lung auscultation revealed the presence of coarse crackles at the left lung base. Intermittent abdominal pain was present in the left lower abdomen.

Laboratory findings were as follows: leukocyte count, $1,100 / \mu \mathrm{L}$ (myelocytes, 2\%; metamyelocytes, $1 \%$; neutrophils, 9\%; eosinophils, 4\%; basophils, 1\%; monocytes, 37\%; and lymphocytes, 46\%); hemoglobin level, $7.7 \mathrm{~g} / \mathrm{dL}$; platelet count, $3.8 \times 10^{4} / \mu \mathrm{L}$; serum albumin, $2.2 \mathrm{~g} / \mathrm{dL}$; lactate dehydrogenase level, $149 \mathrm{U} / \mathrm{L}$; urea nitrogen level, $24 \mathrm{mg} / \mathrm{dL}$; creatinine level, $1.67 \mathrm{mg} / \mathrm{dL}$ (estimated glomerular filtration rate was $24 \mathrm{~mL} / \mathrm{min} / 1.73 \mathrm{~m}^{2}$ ); C-reactive protein level, 35.85 $\mathrm{mg} / \mathrm{dL}$; and IgG level, $824 \mathrm{mg} / \mathrm{dL}$. Liver function test results were nearly within normal ranges. The anti-HIV antibody test result was negative. Chest radiography and plain chest CT revealed the presence of ground-glass opacity in the bilateral lungs and consolidation in the left lower lobe (Fig. 1).

Bacterial pneumonia was suspected, and she was treated with intravenously administered piperacillin/tazobactam (13.5 g; daily). Furthermore, leucovorin administration was commenced for MTX-induced pancytopenia. As the level of serum $\beta$-D-glucan was found to be elevated at $156 \mathrm{pg} / \mathrm{mL}$, PCP was clinically diagnosed, and trimethoprimsulfamethoxazole plus prednisolone ( $80 \mathrm{mg}$; daily) was added to the treatment regimen. On the fifth day after admission, repeated chest radiography and chest CT showed the presence of extensive consolidations in the bilateral lungs, suggesting exacerbation of PCP (Fig. 2). The leukocyte count increased and was found to be $24,200 / \mu \mathrm{L}$ (neutrophils, 59\%; lymphocytes, 13\%) on that day. As worsening of the consolidations coincided with the rapid recovery of the leukocyte count, the presence of IRIS associated with PCP was considered. Methylprednisolone pulse therapy was commenced, and her general conditions improved. The presence of DNA of $P$. jirovecii in the sputum was confirmed by polymerase chain reaction.

On the 6th day after admission, she developed hematochezia. Colonoscopy revealed the presence of a deep punched-out ulcer in the sigmoid colon (Fig. 3). A histopa- 
thological examination of the biopsied mucosa revealed the presence of granulation tissue with inflammatory cell infiltration. An immunohistochemical analysis showed positive results for the CMV antigen. CMV colitis was diagnosed via a macroscopic examination of the colon ulcer and colon histology. Intravenous ganciclovir was administered, and her

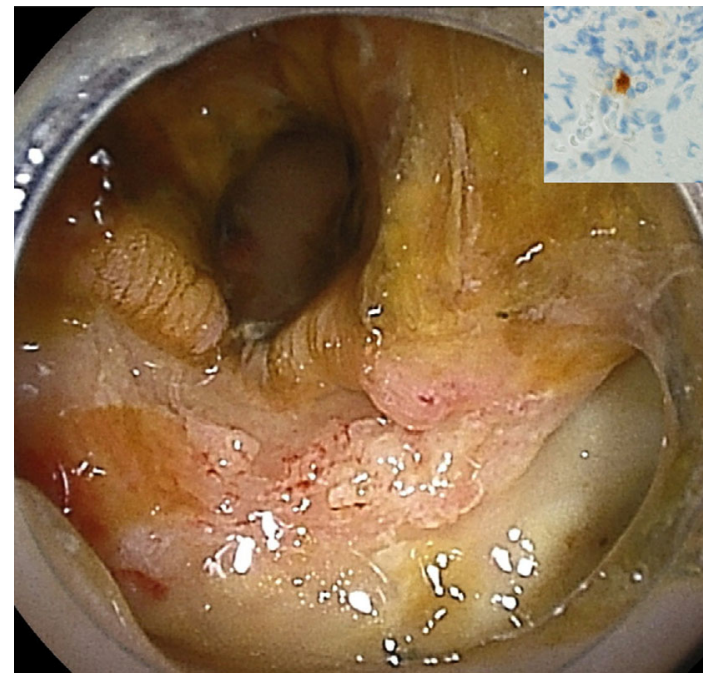

Figure 3. Macroscopic findings of the sigmoid colon on colonoscopy. Immunohistochemical staining demonstrated positivity for cytomegalovirus antigen (Inset). abdominal symptoms subsided. The clinical course of the patient is shown in Fig. 4.

\section{Discussion}

A patient with RA developed IRIS as a manifestation of exacerbated PCP. Rapid recovery of the leukocyte count might have triggered IRIS development. Treatment with methylprednisolone pulse therapy showed improved results. She presented with concomitant CMV colitis, which could also be considered an IRIS.

To our knowledge, IRIS associated with PCP has never been reported in patients who discontinued MTX alone. IRIS associated with tuberculosis has been reported in a patient with RA when both adalimumab and MTX were discontinued; however, the main immunosuppressive agent was adalimumab (9). IRIS associated with PCP shows acute manifestation compared with that triggered by other microorganisms, such as Mycobacterium tuberculosis or Cryptococcus (10). The interval between admission and worsening of the radiography findings was short (five days) in the patient discussed in this report. PCP-IRIS can develop within a week in HIV-positive patients receiving antiviral therapy $(10,11)$. The prognosis of PCP-IRIS is worse in HIVnegative patients than that in HIV-positive patients; indeed, approximately half of non-HIV patients succumbed to PCP,
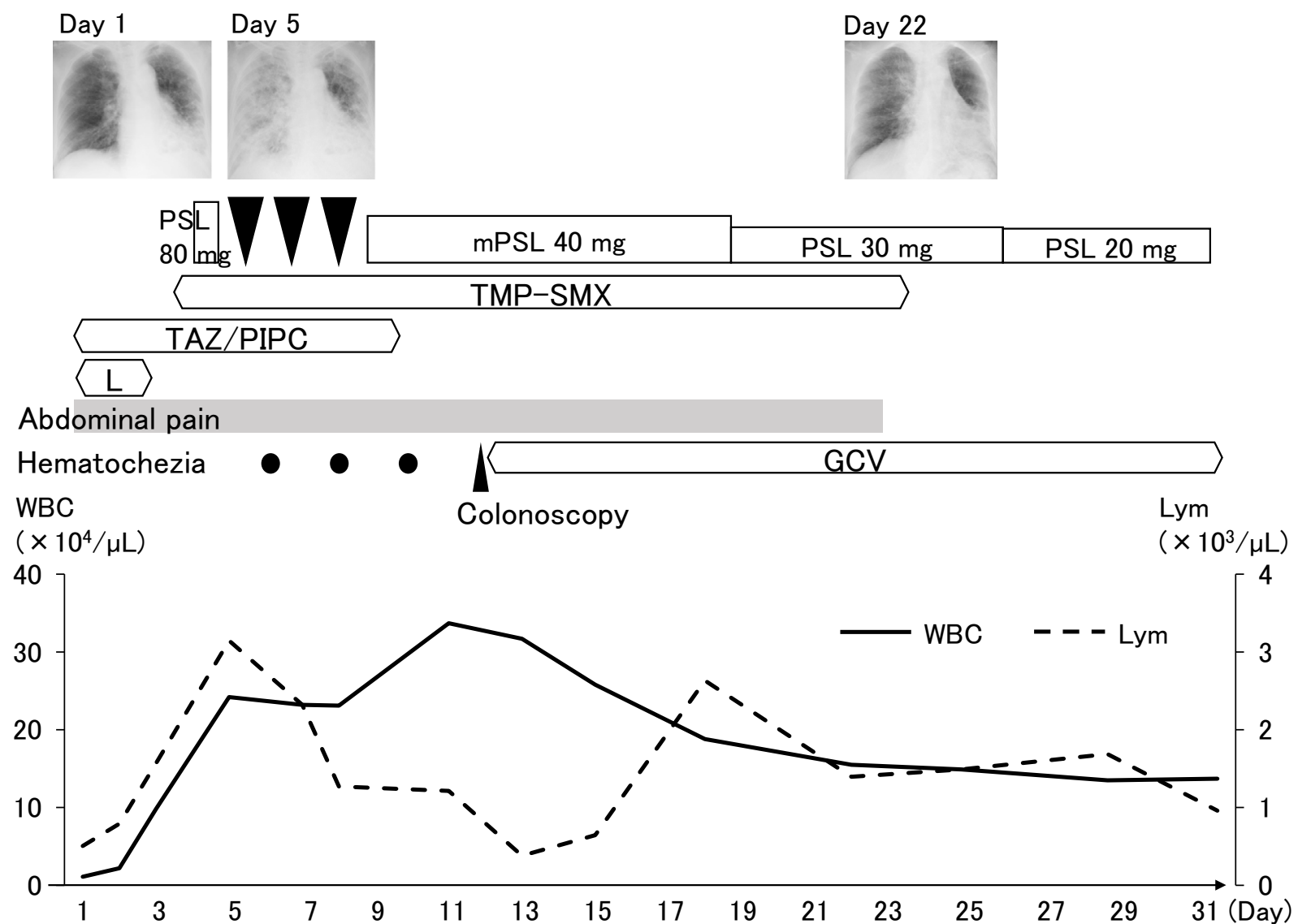

Figure 4. Clinical course of the patient. GCV: ganciclovir, L: Leucovorin, Lym: lymphocytes, mPSL: methylprednisolone, PSL: prednisolone, TAZ/PIPC: tazobactam/piperacillin, TMP-SMX: trimethoprim-sulfamethoxazole, WBC: white blood cells 
whereas PCP-associated death was not reported in HIVpositive patients $(11,12)$. More potent immunosuppressive therapy is required for PCP-IRIS in HIV-negative patients than in HIV-positive patients.

The rapid recovery of the leukocyte count was a trigger for PCP-IRIS development. Regarding IRIS triggered during neutrophil recovery after chemotherapy, invasive aspergillosis and chronic disseminated candidiasis, not PCP, have been reported as causative factors (13). A strong influx of $\mathrm{CD}^{+}$ and $\mathrm{CD}^{+}$cells in the lungs was demonstrated in a patient with HIV after PCP-IRIS had developed (14). In HIVpositive patients, $\mathrm{CD} 4^{+}$cells may be central mediators of IRIS (15). Thus, the rapid recovery of lymphocytes, rather than the recovery of neutrophils, may have contributed to the development of PCP-IRIS in the present patient. The absolute lymphocyte count increased markedly from $506 / \mu \mathrm{L}$ to $3,146 / \mu \mathrm{L}$ on the day after admission. Notably, an increased lymphocyte count is a marker for PCP-IRIS in patients who are HIV-negative immunosuppressed hosts (16). However, as the present patient did not undergo a bronchoalveolar lavage examination, there is no direct evidence that the lymphocytes had accumulated in the lungs.

Furthermore, the marked increase in the lymphocyte count may have exacerbated the CMV colitis. CMV colitis rarely manifests as an IRIS; however, it has been reported as an unmasked IRIS in a patient infected with HIV (17-19). The present patient developed hematochezia, which coincided with the exacerbation of PCP. We speculated that CMV colitis was present before admission, and it markedly manifested along with the rapid recovery of lymphocyte count, causing IRIS development (a paradoxical manifestation of IRIS). One case of CMV-IRIS presenting with a skin ulcer has been reported in a patient with rheumatoid vasculitis with tapering doses of prednisolone and MTX (20).

In conclusion, concurrent development of PCP-IRIS and CMV-IRIS was observed in a patient with RA. The trigger for IRIS development was the rapid recovery of the lymphocyte count from pancytopenia caused by MTX. Since myelosuppression is a major complication observed in patients with RA treated with MTX, clinicians should be alert for IRIS when the lymphocyte count rapidly recovers.

\section{The authors state that they have no Conflict of Interest (COI).}

The patient and her family provided their written informed consent.

\section{References}

1. Gopal R, Rapaka RR, Kolls JK. Immune reconstitution inflammatory syndrome associated with pulmonary pathogens. Eur Respir Rev 26: 2017.

2. Sun HY, Singh N. Immune reconstitution inflammatory syndrome in non-HIV immunocompromised patients. Curr Opin Infect Dis 22: 394-402, 2009.

3. Zheng Y, Zhou H, He Y, Chen Z, He B, He M. The immune pathogenesis of immune reconstitution inflammatory syndrome as- sociated with highly active antiretroviral therapy in AIDS. AIDS Res Hum Retroviruses 30: 1197-1202, 2014.

4. Grant PM, Komarow L, Lederman MM, et al. Elevated interleukin 8 and T-helper 1 and T-helper 17 cytokine levels prior to antiretroviral therapy in participants who developed immune reconstitution inflammatory syndrome during ACTG A5164. J Infect Dis 206: 1715-1723, 2012.

5. Ratnam I, Chiu C, Kandala NB, Easterbrook PJ. Incidence and risk factors for immune reconstitution inflammatory syndrome in an ethnically diverse HIV type 1 -infected cohort. Clin Infect Dis 42: 418-427, 2006.

6. Sueki H, Mizukawa Y, Aoyama Y. Immune reconstitution inflammatory syndrome in non-HIV immunosuppressed patients. J Dermatol 45: 3-9, 2018.

7. Gupta M, Jafri K, Sharim R, et al. Immune reconstitution inflammatory syndrome associated with biologic therapy. Curr Allergy Asthma Rep 15: 499, 2015.

8. Sun HY, Singh N. Opportunistic infection-associated immune reconstitution syndrome in transplant recipients. Clin Infect Dis 53: 168-176, 2011.

9. Wallis RS, van Vuuren C, Potgieter S. Adalimumab treatment of life-threatening tuberculosis. Clin Infect Dis 48: 1429-1432, 2009.

10. Mok HP, Hart E, Venkatesan P. Early development of immune reconstitution inflammatory syndrome related to Pneumocystis pneumonia after antiretroviral therapy. Int J STD AIDS 25: 373-377, 2014.

11. Wu AK, Cheng VC, Tang BS, et al. The unmasking of Pneumocystis jiroveci pneumonia during reversal of immunosuppression: case reports and literature review. BMC Infect Dis 4: 57, 2004.

12. Roade Tato L, Burgos Cibrian J, Curran Fabregas A, et al. Immune reconstitution inflammatory syndrome in HIV-infected patients with Pneumocystis jirovecii pneumonia. Enferm Infecc Microbiol Clin 36: 621-626, 2018.

13. Dellière $S$, Guery R, Candon $S$, et al. Understanding Pathogenesis and Care Challenges of Immune Reconstitution Inflammatory Syndrome in Fungal Infections. J Fungi (Basel) 4: 2018.

14. Barry SM, Lipman MC, Deery AR, Johnson MA, Janossy G. Immune reconstitution pneumonitis following Pneumocystis carinii pneumonia in HIV-infected subjects. HIV Med 3: 207-211, 2002.

15. Barber DL, Andrade BB, Sereti I, Sher A. Immune reconstitution inflammatory syndrome: the trouble with immunity when you had none. Nat Rev Microbiol 10: 150-156, 2012.

16. Cheng VC, Hung IF, Wu AK, Tang BS, Chu CM, Yuen KY. Lymphocyte surge as a marker for immunorestitution disease due to Pneumocystis jiroveci pneumonia in HIV-negative immunosuppressed hosts. Eur J Clin Microbiol Infect Dis 23: 512-514, 2004.

17. von Both U, Laffer R, Grube C, Bossart W, Gaspert A, Günthard HF. Acute cytomegalovirus colitis presenting during primary HIV infection: an unusual case of an immune reconstitution inflammatory syndrome. Clin Infect Dis 46: e38-e40, 2008.

18. Yoshida S, Mori N, Honda M. Cytomegalovirus colitis in a patient with HIV infection shortly after initiation of antiretroviral therapy. IDCases 17: e00552, 2019.

19. Alukal J, Asif M, Mundada R, McNamee WB. Recurrent cytomegalovirus colitis: a rare case of immune reconstitution inflammatory syndrome. BMJ Case Rep 2018: 2018.

20. Katsuta M, Shiohara T, Asahina A. Refractory rheumatoid vasculitis complicated by cytomegalovirus reactivation as a manifestation of immune reconstitution inflammatory syndrome. JAAD Case Rep 6: 652-655, 2020.

The Internal Medicine is an Open Access journal distributed under the Creative Commons Attribution-NonCommercial-NoDerivatives 4.0 International License. To view the details of this license, please visit (https://creativecommons.org/licenses/ by-nc-nd/4.0/). 
(C) The Japanese Society of Internal Medicine Intern Med Advance Publication 